\title{
Integer partitions with fixed subsums
}

\author{
Yu. Yakubovich* \\ Department of Mathematics \\ Utrecht University \\ P.O.box 80010 \\ NL-3508 TA Utrecht \\ The Netherlands \\ yakubovichmath.uu.nl
}

Submitted: Jan 17, 2005; Accepted: May 11, 2005; Published: May 16, 2005

Mathematics Subject Classifications: 05A17

\begin{abstract}
Given two positive integers $m \leq n$, we consider the set of partitions $\lambda=$ $\left(\lambda_{1}, \ldots, \lambda_{\ell}, 0, \ldots\right), \lambda_{1} \geq \lambda_{2} \geq \ldots$, of $n$ such that the sum of its parts over a fixed increasing subsequence $\left(a_{j}\right)$ is $m: \lambda_{a_{1}}+\lambda_{a_{2}}+\cdots=m$. We show that the number of such partitions does not depend on $n$ if $m$ is either constant and small relatively to $n$ or depend on $n$ but is close to its largest possible value: $n-m a_{1}=k$ for fixed $k$ (in the latter case some additional requirements on the sequence $\left(a_{j}\right)$ are needed). This number is equal to the number of so-called colored partitions of $m$ (respectively $k$ ). It is proved by constructing bijections between these objects.
\end{abstract}

\section{Introduction}

In a recent paper [2] Canfield and his collaborators considered a set of partitions $\lambda=$ $\left(\lambda_{1}, \lambda_{2}, \ldots\right), \lambda_{1} \geq \lambda_{2} \geq \ldots$, of an integer $n$ with a fixed sum of even parts (i.e. $\lambda_{2}+$ $\lambda_{4}+\cdots=m$ ). They, in particular, proved that the number of such partitions depends only on $m$ for sufficiently large $n$ (namely, for $n \geq 3 m$ ) and equals to the number of colored partitions of $m$. These are partitions of $m$ with each part having an additional attribute, usually referred to as "color", which can take two values in this particular case. The number of such partitions $f(m)$ is well known, and the generating function for these numbers is

$$
\sum_{m \geq 0} f(m) x^{m}=\prod_{k \geq 1} \frac{1}{\left(1-x^{k}\right)^{2}},
$$

*This research was supported by the NWO postdoctoral fellowship. 
see, e.g. [1]. In other words, as mentioned in [2], these numbers count ordered pairs of partitions $(\lambda, \mu)$ such that $|\lambda|+|\mu|=m$. Their proof is based on construction of a bijection between the set of partitions of $n$ with the sum of even parts being equal to $m$ and a pairs of partitions $(\lambda, \mu)$ where $|\lambda|+|\mu|=m$ and $\mu$ has at most $n-2 m$ parts.

We generalize this result in the following way. Let $\left(a_{j}\right)_{j \geq 0}$ be a strictly increasing sequence of positive integers. Denote by $\mathcal{P}_{\left(a_{j}\right)}(n, m)$ the set of partitions $\lambda$ of $n$ such that $\sum_{j \geq 0} \lambda_{a_{j}}=m$. Then the following statement holds:

Theorem 1. Let $\left(a_{j}\right)_{j \geq 0}$ be a strictly increasing integer sequence with $a_{0}=2$. Then there exists a function $N(m)$ such that for all $n \geq N(m)$ we have $\# \mathcal{P}_{\left(a_{j}\right)}(n, m)=q(m)$ where the generating function for $q(m)$ is given by

$$
\sum_{m \geq 0} q(m) x^{m}=\prod_{j \geq 1} \frac{1}{\left(1-x^{j}\right)^{b_{j}}}, \quad b_{j}=a_{j}-a_{j-1} .
$$

The number $q(m)$ can be also described as a number of colored partitions of $m$ with $b_{j}$ possible colors for parts $j$. We call these partitions $\left(b_{j}\right)_{j \geq 1}$-colored partitions. The result of [2] is a particular case of this theorem for the sequence $a_{j}=2 j+2$. We shall prove this statement in Section 2.

Further investigation of sequences $\# \mathcal{P}_{\left(a_{j}\right)}(n, m)$ for regular $\left(a_{j}\right)$ shows that they have another stabilization property. An obvious observation that

$$
\# \mathcal{P}_{(2 j+2)}(n, m)=\# \mathcal{P}_{(2 j+1)}(n, n-m)
$$

suggests an idea that for some sequences $\left(a_{j}\right)$ the stabilization should take place from the end of the sequence. (We use a notation $(2 j+2)$ for a sequence which has a common term $2 j+2$; it is always supposed that $j=0,1,2, \ldots$ unless explicitly specified.) Let $\left(d_{j}\right)_{j \geq 0}$ be an integer sequence with the following properties: (i) $d_{0} \geq 1$, (ii) $d_{1}>2 d_{0}$ and $d_{j}-d_{j-1} \geq d_{0}$ for $j>1$, and (iii) $d_{j}-j d_{0} \rightarrow+\infty$. Given $\left(d_{j}\right)$ and an integer $n$ we consider a sequence $\# \mathcal{P}_{\left(d_{j}\right)}(n, m)$ and notice that the last nonzero term in it occurs for $m=\left[n / d_{0}\right]$. Indeed, if $\lambda \in \mathcal{P}_{\left(d_{j}\right)}(n, m)$ then

$$
n=\sum_{j \geq 1} \lambda_{j} \geq d_{0} \sum_{j \geq 1} \lambda_{j d_{0}} \geq d_{0} \sum_{j \geq 0} \lambda_{d_{j}}=m d_{0},
$$

because $d_{j} \geq(j+1) d_{0}$ by properties (i) and (ii), and for $m=\left[n / d_{0}\right]$ there exist at least one partition $\left(n-\left(d_{0}-1\right) m, m, \ldots, m\right) \in \mathcal{P}_{\left(d_{j}\right)}(n, m)$ with exactly $d_{0}$ nonzero parts. However it turns out that for $d_{0}>1$ the stabilization takes place periodically in the following sense: $\# \mathcal{P}_{\left(d_{j}\right)}\left(n,\left[n / d_{0}\right]-m\right)$ depends only on a residue of $n\left(\bmod d_{0}\right)$ for large $n$. In other words, the sequence $\# \mathcal{P}_{\left(d_{j}\right)}\left(n d_{0}+m, n\right)$ does not depend on $n$ for large $n$.

To make a precise statement let us consider a mapping which sends the sequence $\left(d_{j}\right)_{j \geq 0}$ satisfying properties (i)-(iii) to a sequence $\left(b_{j}\right)_{j \geq 1}$ by the following rule:

$$
b_{j}=\sup \left\{i: d_{i}^{\prime} \leq j\right\}+2-\inf \left\{i \geq 0: d_{i}^{\prime}>j-d_{0}\right\}, \quad d_{i}^{\prime}=d_{i}-(i+1) d_{0} .
$$

First note that the definition is correct because $d_{i}^{\prime}$ is a nondecreasing sequence growing to $\infty$ by properties (ii) and (iii) and thus $b_{j}$ is finite. Next, note that $b_{j}>0$. Indeed, $\sup \left\{i: d_{i}^{\prime} \leq j\right\}+1 \geq \inf \left\{i: d_{i}^{\prime} \geq j\right\} \geq \inf \left\{i: d_{i}^{\prime}>j-d_{0}\right\}$. 
Theorem 2. Let $\left(d_{j}\right)_{j \geq 0}$ be an integer sequence satisfying properties (i)-(iii). Then there exists a function $N(m)$ such that for all $n \geq N(m)$ we have $\# \mathcal{P}_{\left(d_{j}\right)}\left(n d_{0}+m, n\right)=q(m)$, and the generating function for sequence $q(m)$ is given by

$$
\sum_{m \geq 0} q(m) x^{m}=\prod_{j \geq 1} \frac{1}{\left(1-x^{j}\right)^{b_{j}}}
$$

where $b_{j}$ is defined by (1).

Actually, Theorem 1 is a corollary of Theorem 2 . To see this, consider a sequence $\left(a_{j}\right)$ with $a_{0}=2$ and take a sequence $d_{j}$ going through all numbers in $\mathbb{N} \backslash\left\{a_{j}\right\}_{j \geq 0}$ in increasing order. Clearly, $d_{0}=1$ and properties (i)-(iii) are satisfied. The sequence $d_{j}^{\prime}$ starts with 0 followed by $a_{1}-a_{0}-1$ terms 1 followed by $a_{2}-a_{1}-1$ terms 2 etc. It is easy to see that $d_{j}=i$ when $a_{i-1}-i \leq j \leq a_{i}-i-2$. Calculation of $b_{j}$ by formula (1) shows that $b_{j}=a_{j}-a_{j-1}$ and thus Theorem 1 holds.

Note that the mapping from a sequence $(q(m))$ to the sequence $\left(b_{j}\right)$ is known as the inverse Euler transform, see [3, p. 20-21]. Some of the sequences $q(m)$ corresponding to a regular sequences $\left(b_{j}\right)$ appear also at the online Encyclopedia of integer sequences, [4].

It is my pleasure to thank the anonymous referee for pointing out some misprints in the original text.

\section{Proof of Theorem 1}

It was noted after Theorem 2 that it implies Theorem 1. However we prefer to give a direct proof of Theorem 1 . While proofs of both theorems are bijective and the bijection is essentially the same, its description is significantly simplified for a special case $d_{0}=1$.

The direct proof of Theorem 1 is very easy and consists of an explicit construction of a bijection between the $\mathcal{P}_{\left(a_{j}\right)_{j \geq 0}}(n, m)$ with $a_{0}=2$ and $\left(b_{j}\right)_{j \geq 1}$-colored partitions of $m$ with $b_{j}=a_{j}-a_{j-1}$. Let $\lambda \in \mathcal{P}_{\left(a_{j}\right)_{j}>0}(n, m)$ be such partition. Note that we already have a partition of $m$, namely $\mu=\lambda_{\left(a_{j}\right)}=\left(\lambda_{a_{0}}, \lambda_{a_{1}}, \lambda_{a_{2}}, \ldots\right)$. So it seems that all we have to do is just to specify the color of each part.

But it is not exactly what we are going to do. In fact we are going to color a partition $\mu^{\prime}$ conjugate to $\mu$, i.e. the partition $1^{\mu_{1}-\mu_{2}} 2^{\mu_{2}-\mu_{3}} \ldots m^{\mu_{m}-\mu_{m+1}}$. Here $1^{k_{1}} 2^{k_{2}} \ldots m^{k_{m}}, k_{i} \geq 0$, denotes the partition of $m$ having $k_{i}$ parts equal to $i$; obviously, $\sum_{i} i k_{i}=m$. Now we are ready to color the parts of $\mu^{\prime}$. Take a part of size $j$, there are exactly $\mu_{j}-\mu_{j+1}=\lambda_{a_{j-1}}-\lambda_{a_{j}}$ such parts which should be colored in $b_{j}=a_{j}-a_{j-1}$ colors. Let us number these colors by $1, \ldots, b_{j}$. For each $c \in\left\{1, \ldots, b_{j}\right\}$ take exactly $\lambda_{a_{j-1}+c-1}-\lambda_{a_{j-1}+c}$ parts of color $c$. This number is non-negative because $\lambda$ is a partition. At the same time there are

$$
\left(\lambda_{a_{j-1}}-\lambda_{a_{j-1}+1}\right)+\left(\lambda_{a_{j-1}+1}-\lambda_{a_{j-1}+2}\right)+\cdots+\left(\lambda_{a_{j-1}+b_{j}-1}-\lambda_{a_{j-1}+b_{j}}\right)=\lambda_{a_{j-1}}-\lambda_{a_{j}}
$$

parts of size $j$ because $a_{j-1}+b_{j}=a_{j}$ by definition. Thus it is a correctly defined mapping from $\mathcal{P}_{\left(a_{j}\right)_{j \geq 0}}(n, m)$ to $\left(b_{j}\right)$-colored partitions. 
It is clear that the constructed mapping is injective. To show that it is a surjection let us try to find a preimage of a given $\left(b_{j}\right)$-colored partition. It turns out that it is always possible for large $n$. Let a sequence $\left(b_{j}\right)$ be fixed and let $a_{0}=2$ and $a_{j}=2+b_{1}+\cdots+b_{j}$ for $j \geq 1$. Take a $\left(b_{j}\right)$-colored partition $\mu$ of $m$; it can be parameterized by numbers $k_{j, c}$ $\left(j=1, \ldots, m\right.$ and $\left.c=1, \ldots, b_{j}\right)$ which denote the number of parts of size $j$ and of color $c$ in the partition $\mu$. We denote $k_{j}=\sum_{c=1}^{b_{j}} k_{j, c}$; since $\mu \vdash m$ we have $\sum_{j=1}^{m} j k_{j}=m$. The preimage of the partition $\mu$ under the mapping described above can be written as follows:

$$
\lambda_{i}=\sum_{j \geq j_{0}} k_{j}+\sum_{c=i-a_{j_{0}-1}+1}^{b_{j_{0}}} k_{j_{0}-1, c} \quad \text { for } i \in\left[a_{j_{0}-1}, a_{j_{0}}\right), \quad j_{0} \geq 1
$$

(To say it in words, if $a_{j_{0}-1} \leq i<a_{j_{0}}$ then $\lambda_{i}$ is the number of parts of $\mu$ of any color and size not less than $j_{0}$, plus the number of parts of size $j_{0}-1$ having colors $i-a_{j_{0}-1}+1, \ldots, b_{j_{0}}$.) This way we define all parts of $\lambda$ but $\lambda_{1}$. The latter can be defined from $\lambda_{1}=n-\left(\lambda_{2}+\lambda_{3}+\ldots\right)$ but we need to verify that $\lambda_{1} \geq \lambda_{2}$. It is clear from (2) that for a given partition $\mu$ the maximal value of $\lambda_{2}+\lambda_{3}+\ldots$ is achieved if $k_{j, c}=0$ for $c<b_{j}$ and $k_{j, b_{j}}=k_{j}$ and is equal to $k_{1} b_{1}+k_{2}\left(b_{1}+b_{2}\right)+\ldots$ Since $\lambda_{2}=k_{1}+k_{2}+\ldots$ we see that if

$$
n \geq N(m)=\max _{\mu \vdash m} k_{1}(\mu)\left(1+b_{1}\right)+k_{2}(\mu)\left(1+b_{1}+b_{2}\right)+\ldots
$$

then $\lambda_{1} \geq \lambda_{2}$ for all $\mu \vdash m$ and thus our mapping is a bijection.

Remark. It seems to be difficult to find an explicit formula for $N(m)$ but its value for a given sequence $\left(b_{j}\right)$ can be easily found using linear programming algorithms. For some degenerate sequences $\left(b_{j}\right)$ it can be found explicitly. For instance, if $a_{j}=2+d j$ is an arithmetic progression, it can be easily seen that $N(m)=(d+1) m$.

\section{Proof of Theorem 2}

We again construct a bijection between partitions in $\mathcal{P}_{\left(d_{j}\right)_{j \geq 0}}(n k+m, n)$ and $\left(b_{j}\right)$-colored partitions with $b_{j}$ defined by (1). In order to do it we introduce the mapping $s: \mathbb{N} \rightarrow \mathbb{Z}_{+}$ by

$$
s(i)=i-j d_{0}, \quad \text { for } d_{j-1} \leq i<d_{j}, \quad j \geq 0 ;
$$

here and below we suppose $d_{-1}=0$. Clearly, the mapping $s$ depends on a sequence $\left(d_{j}\right)$. First we prove the following simple result.

Lemma 1. The only number which is mapped to 0 by $s$ is $d_{0}$. For $j \geq 1$, exactly $b_{j}$ natural numbers are mapped to $j$ by $s$, namely

$$
\{i: s(i)=j\}=\left\{i_{1}, i_{2}, \ldots, i_{b_{j}}\right\}
$$

where $d_{j_{0}-2+c} \leq i_{c}<d_{j_{0}-1+c}$ and $j_{0}=\inf \left\{i>0: d_{i}^{\prime}>j-d_{0}\right\}$. 
Proof. Let us take a look on the sequence $(s(i))_{i>1}$. It starts with 1 , increases by 1 to $s\left(d_{0}-1\right)=d_{0}-1$, then jumps backwards to $s\left(d_{0}\right)=0$ and increases by 1 to $s\left(d_{1}-1\right)=$ $d_{1}-1-d_{0}$, then jumps backwards to $s\left(d_{1}\right)=d_{1}-2 d_{0}$, etc. It is quite clear that property (ii) of $\left(d_{j}\right)$ implies that $s\left(d_{j}\right) \geq s\left(d_{j-1}\right)$ and $s\left(d_{j}-1\right) \geq s\left(d_{j-1}-1\right)$ for $j \geq 2$. Thus, while the sequence $(s(i))$ is not monotone, it has the following properties: if $i \geq d_{j}$ for some $j \geq 0$ then $s(i) \geq s\left(d_{j}\right)$ and if $i \leq d_{j}-1$ then $s(i) \leq s\left(d_{j}-1\right)$.

Since by property (ii) $s\left(d_{1}\right)>s\left(d_{0}\right)=0, s(i)=0$ implies $i=d_{0}$ and the first assertion holds. Next, the sequence $s(i)$ either weakly decreases or increases by 1 , and goes to $\infty$; consequently, the set $s^{-1}(k)=\{i: s(i)=k\}$ is not empty for all $k \geq 1$. Take some $k \geq 1$ and consider $i_{1}=\min s^{-1}(k)$. Let $j_{1}$ be such that $d_{j_{1}-1} \leq i_{1}<d_{j_{1}}$; if $d_{0}=1$ then $j_{1} \geq 1$ and if $d_{0}>1$ then $j_{1} \geq 0$. Clearly, $s(i)<k$ for all $i<i_{1}$, and $k \leq s\left(d_{j_{1}}-1\right)$. Thus $j_{1}=\inf \left\{j: s\left(d_{j}-1\right) \geq k\right\}=\inf \left\{j: d_{j}^{\prime}>k-d_{0}\right\}$.

Now let $i_{\max }=\max s^{-1}(k)$ (it exists since $s(i)$ grows to infinity) and let $j_{\max }$ be such that $d_{j_{\max }-1} \leq i_{\max }<d_{j_{\max }}$. Then

$$
s\left(d_{j_{\max }-1}\right)=d_{j_{\max }-1}-j_{\max } d_{0} \leq k<d_{j_{\max }}-\left(j_{\max }+1\right) d_{0}=s\left(d_{j_{\max }}\right)
$$

and $j_{\max }-1=\max \left\{j: d_{j}^{\prime} \leq k\right\}$. For each $j$ satisfying $j_{1} \leq j \leq j_{\text {max }}$ there exists exactly one $i \in\left[d_{j-1}, d_{j}\right)$ such that $s(i)=k$. Indeed, $s\left(d_{j-1}\right) \leq k \leq s\left(d_{j}-1\right)$ and $s(i)$ is increasing by 1 on this interval.

Now we are ready to construct a required bijection. Let us start with a partition $\lambda \in \mathcal{P}_{\left(d_{j}\right)}\left(n d_{0}+m, n\right)$. Consider a conjugate partition $\lambda^{\prime}$ which is $1^{\lambda_{1}-\lambda_{2}} 2^{\lambda_{2}-\lambda_{3}} \ldots\left(n d_{0}+\right.$ $m)^{\lambda_{n d_{0}+m}-\lambda_{n d_{0}+m+1}}$. We are going to decrease some parts of $\lambda^{\prime}$ in order to get a partition $\mu$ of $m$. However several parts of $\mu$ of the same size can originate from parts of $\lambda^{\prime}$ of different sizes, and we are coloring them in different colors to keep track of their origin and make the mapping reversible.

To be more precise, let us transform each part of size $i$ in $\lambda^{\prime}$ into a part of size $s(i)$. First, we claim that the sum of transformed parts will be exactly $m$. Indeed, for $j \geq 0$ all parts of size $i$ satisfying $d_{j} \leq i<d_{j+1}$ are transformed in $i-(j+1) d_{0}$, and there are $\lambda_{d_{j}}-\lambda_{d_{j+1}}$ such parts. Thus the total number subtracted from the sum $n d_{0}+m$ is

$$
d_{0} \sum_{j \geq 0}(j+1)\left(\lambda_{d_{j}}-\lambda_{d_{j+1}}\right)=d_{0} \sum_{j \geq 0} \lambda_{d_{j}}=n d_{0}
$$

and sum of transformed parts is $m$.

Second, according to Lemma 1 there are exactly $b_{j}$ different part sizes which are transformed into $j$. If we color each part in one of $b_{j}$ colors according to the size of original part we can restore the original partition $\lambda^{\prime}$ from its image, the colored partition of $m$.

So we constructed a mapping from $\mathcal{P}_{\left(d_{j}\right)_{j \geq 0}}\left(n d_{0}+m, n\right)$ to $\left(b_{j}\right)$-colored partitions. It is injective, since we can easily invert the transformation described above knowing the correspondence between colors of part $j$ and parts of $\lambda^{\prime}$ in $s^{-1}(j)$. However it is not surjective for $m$ relatively large compared to $n$. The reason of this is the existance of 
parts of size $d_{0}$ in $\lambda^{\prime}$ which were transformed to 0 and then silently neglected. Their number can be determined from the condition

$$
\sum_{j \geq 0} \lambda_{d_{j}}=n
$$

but it might happen for large $m$ that their quantity should be negative, that certainly cannot take place.

Let $\mu$ be a $\left(b_{j}\right)$-colored partition; we parameterize it by numbers $k_{j, c}, j=1, \ldots, m$ and $c=1, \ldots, b_{j}$, each $k_{j, c}$ denoting the number of parts of size $j$ and color $c$ in $\mu$. Let $s_{c}^{-1}(j)$ denote $i_{c}$ from Lemma 1 , i.e. the number such that $s\left(i_{c}\right)=j$ and $d_{j_{1}+c-2} \leq i_{c}<d_{j_{1}+c-1}$ where $j_{1}=\inf \left\{i>0: d_{i}^{\prime}>j-d_{0}\right\}$. Then parts of the original partition $\lambda$ can be reconstructed from $\mu$ as

$$
\lambda_{i}=\sum_{(j, c): s_{c}^{-1}(j) \geq i} k_{j, c}+k_{0} 1\left(i \leq d_{0}\right),
$$

where $k_{0}$ is the (unknown yet) number of parts $d_{0}$ in $\lambda^{\prime}$. Thus

$$
n=\sum_{i \geq 0} \lambda_{d_{i}}=k_{0}+\sum_{(j, c)}\left(j_{\max }(j)+c-1\right) k_{j, c}
$$

since $k_{j, c}$ occurs as a summand in $\lambda_{d_{i}}$ only for $i<j_{\max }(j)+c-1$. On the other hand, $\sum_{(j, c)} j k_{j, c}=m$, and so the sum in the RHP of (5) is bounded from above (as a function of $k_{j, c}$ 's) by some function of $m$, say $N(m)$. If $n \geq N(m)$ then $k_{0}$ is not negative for any choice of a colored partition $\mu$, and thus our mapping is bijection.

An interesting fact is that if $d_{j}=h+2 j h$ then the sequence $\left(b_{j}\right)$ derived from it by $(1)$ does not depend on $h$ (namely, all $b_{j}=2$ ). It might be instructive to describe a direct bijection between $\mathcal{P}_{(h+2 j h)_{j \geq 0}}(h n+m, n)$ and $\mathcal{P}_{(p+2 j p)_{j \geq 0}}(p n+m, n)$ for $h \neq p$.

\section{References}

[1] G. Andrews. The Theory of Partitions. Encyclopedia of mathematics and its applications, Vol. 2. Addison-Wesley, 1976.

[2] E. R. Canfield, C. D. Savage, H. Wilf. Regularly spaced subsums of integer partitions. Acta Arithmetica, 115 (2004), no. 3, 205-216; preprint version available at arXiv:math.CO/0308061.

[3] N. J. A. Sloane and S. Plouffe. The Encyclopedia of Integer Sequences. Academic Press, San Diego, 1995, 587 pp.

[4] N. J. A. Sloane. The On-Line Encyclopedia of Integer Sequences, on the web at http://www.research.att.com/ njas/sequences/. 